\title{
Lentinula edodes mycelia extract plus adjuvant chemotherapy for breast cancer patients: Results of a randomized study on host quality of life and immune function improvement
}

\author{
YUKIKO NAGASHIMA ${ }^{1}$, SHIGEHUMI YOSHINO ${ }^{2}$, SHIGERU YAMAMOTO ${ }^{3}$, NORIKO MAEDA $^{3}$, \\ TATSUYA AZUMI ${ }^{3}$, YOSHIFUMI KOMOIKE ${ }^{4}$, KIYOTAKA OKUNO ${ }^{4}$, TSUTOMU IWASA $^{5}$, JUNJI \\ TSURUTANI $^{5}$, KAZUHIKO NAKAGAWA ${ }^{5}$, OKA MASAAKI ${ }^{6}$ and NAGANO HIROAKI ${ }^{3}$
}

\begin{abstract}
${ }^{1}$ Department of Breast and Thyroid Surgery, Japan Community Health Care Organization (JCHO) Shimonoseki Medical Center, Shimonoseki, Yamaguchi 750-0061; ${ }^{2}$ Oncology Center, Yamaguchi University Hospital, ${ }^{3}$ Department of Gastroenterological,

Breast and Endocrine Surgery, Yamaguchi University Graduate School of Medicine, Ube, Yamaguchi 755-0046; Departments of ${ }^{4}$ Surgery and ${ }^{5}$ Medical Oncology, Kindai University School of Medicine, Osakasayama, Osaka 589-8511; ${ }^{6}$ Yamaguchi University, Yamaguchi 753-8511, Japan
\end{abstract}

Received February 10, 2017; Accepted July 22, 2017

DOI: $10.3892 /$ mco.2017.1346

\begin{abstract}
Anthracycline-based chemotherapies for breast cancer are known to adversely affect patients' quality of life (QOL) and immune function. For that reason, adjuvants that improve those impairments are required. A randomized double-blind study was conducted to evaluate the effectiveness of Lentinula edodes mycelia extract (LEM), which is an oral biological response modifier (BRM) medicine for cancer patients as such an adjuvant. A total of 47 breast cancer patients who were scheduled to receive postoperative adjuvant anthracycline-based chemotherapy, i.e., 5-fluorouracil (5-FU) + cyclophosphamide + epirubicin (FEC regimen), 5-FU + cyclophosphamide + doxorubicin/pirarubicin (FAC regimen), cyclophosphamide + doxorubicin/pirarubicin (AC regimen) and cyclophosphamide + epirubicin (EC regimen), were entered in the study. The patients were randomly divided into either an LEM or a placebo tablet group; the tablets were orally ingested daily over 2 courses of each therapy. In the placebo group, the total scores for QOL were lower on day 8 of the second course of chemotherapy compared with the baseline scores, whereas in the LEM group the scores had not decreased. In the placebo group, the QOL functional well-being score was lower on day 8 after both the first and second courses of chemotherapy compared with the baseline score, but it had not decreased in the LEM group. Evaluation of immunological parameters
\end{abstract}

Correspondence to: Dr Yukiko Nagashima, Department of Breast and Thyroid Surgery, Japan Community Health Care Organization (JCHO) Shimonoseki Medical Center, 3-3-8 Kami-shinchi-cho, Shimonoseki, Yamaguchi 750-0061, Japan

E-mail: yukikonagashima@outlook.jp

Key words: randomized clinical study, breast cancer, anthracycline, Lentinula edodes, regulatory $\mathrm{T}$ cell, quality of life indicated that an increase in the proportion of regulatory $\mathrm{T}$ cells to peripheral blood $\mathrm{CD}^{+}$cells tended to be inhibited in the LEM group compared with the placebo group. Oral LEM that was coadministered with anthracycline-based chemotherapies was useful for maintaining patients' QOL and immune function. Thus, LEM appears to be a useful oral adjuvant for patients receiving anthracycline-based chemotherapy.

\section{Introduction}

An increasing proportion of patients with early-stage breast cancer are treated with taxanes as postoperative adjuvant chemotherapy. Anthracycline-based chemotherapies are effective and are also widely used as the main adjuvant chemotherapies (1-3); however, those treatments are associated with well-known adverse reactions, such as hematotoxicity, nausea, vomiting and hair loss $(4,5)$. The use of 5HT3 receptor antagonists allows some degree of control of the symptoms of late-onset nausea and vomiting (6-8). However, patients on anthracycline-based chemotherapy experience fatigue and a decrease in their activity and daily quality of life (QOL), and these problems may be difficult to control (9-11). Moreover, although the host immune function of breast cancer patients on chemotherapy affects the recurrence rate (12), anthracycline-based chemotherapy is reported to adversely affect immune function (13). Accordingly, there is a need for treatments that can maintain and/or improve the QOL and immune function of breast cancer patients receiving anthracycline-based chemotherapy.

Lentinula edodes mycelia extract (LEM) is a dried powder of a hot water extract of the mycelia of Lentinula edodes (14). Oral LEM demonstrated in vivo activity in promoting antitumor effect and improving host immune function (14-19). In gastrointestinal cancer patients undergoing chemotherapy, oral LEM exerted an inhibitory effect on the incidence of adverse events (AEs), such as chemotherapy-induced nausea, and also improved immune function, by increasing natural killer 
(NK) cell activity, resulting in improvement of the patients' QOL $(20,21)$. LEM also improved the QOL and immune function of breast cancer patients undergoing postoperative adjuvant hormone therapy (22).

The results of a study on breast cancer patients who were administered 5 -fluorouracil $(5-\mathrm{FU})$ at $500 \mathrm{mg} / \mathrm{m}^{2}$, cyclophosphamide at $500 \mathrm{mg} / \mathrm{m}^{2}$ and epirubicin at $75 \mathrm{mg} / \mathrm{m}^{2}$ (FEC75 regimen) every 3 weeks as postoperative adjuvant chemotherapy were previously reported (23). In that study, oral LEM was co-administered during the second course of chemotherapy and the QOL and immune function of the second course were compared to those of the first course. The results demonstrated that the decrease in QOL as estimated by the QOL Questionnaire for Cancer Patients Treated with Anticancer Drugs (QOL-ACD) (24) and the decrease in NK cell activity caused by the FEC75 regimen were improved by the co-administration of LEM in the second course of the chemotherapy compared with the first course, demonstrating the usefulness of LEM. However, that study was a single-group open trial, and administration of LEM was limited to the 3 weeks of the second course of chemotherapy. Due to that design, the possibility that the improvement seen during the second course was due to the patients becoming accustomed to the chemotherapy cannot be ruled out. Moreover, at the time of that study, the use of 5HT3 receptor antagonists for nausea and vomiting was not yet commonplace, and they were not used in the study. Therefore, it is not clear whether the improving effect of LEM on the QOL could also be achieved with the currently widely used 5HT3 receptor antagonists. For those reasons, the present placebo-controlled randomized double-blind study was designed and conducted to evaluate the effectiveness of LEM in improving the QOL and the immune function and controlling the AEs caused by postoperative adjuvant anthracycline-based chemotherapy administered to early-stage breast cancer patients who were also administered 5HT3 receptor antagonists as supportive therapy.

\section{Patients and methods}

Patients. In the present study, patients who met the following criteria were enrolled: The patients were female, aged $\geq 20$ years, were diagnosed with breast cancer, and were scheduled for anthracycline-based adjuvant chemotherapy as postoperative adjuvant therapy. The patients had maintained principal organ function for chemotherapy, with a performance status of 0 or 1 , and had a life expectancy of $>3$ months. Patients who were pregnant, were at risk of becoming pregnant, were breastfeeding or had received prior treatment within 4 weeks of the entry to this study, were excepted. All the patients were fully informed on the aims and methods of the trial prior to participation and provided written consent for participation in the trial.

Drug. The LEM used was manufactured by Kobayashi Pharmaceutical Co. Ltd. (Osaka, Japan). The manufacturing process was as previously reported (14). Briefly, Lentinula edodes mycelia (strain NITE SD 0043, registered at the National Institute of Technology and Evaluation in Japan) were first inoculated onto solid medium consisting mainly of bagasse of sugarcane and defatted rice polishings, and cultured until the mycelia had spread. The culture was then extracted using hot water. The dissolved component was dried to obtain a dry powder. This dry powder was used as the LEM in the present trial. The LEM group patients were dispensed 230-mg test tablets that contained $150 \mathrm{mg}$ of LEM, and were instructed to ingest 6 tablets in the morning and another 6 in the evening. The placebo group patients were dispensed $230-\mathrm{mg}$ placebo tablets that contained caramel color, dextrin and maltitol, and were instructed to ingest 6 tablets in the morning and 6 in the evening. Both the test and the placebo tablets were manufactured by Kobayashi Pharmaceutical Co. Ltd.

Study design. This was a placebo-controlled randomized double-blind study that evaluated the effectiveness of LEM during the first 2 courses of adjuvant anthracycline-based chemotherapy administered as postoperative adjuvant therapy (UMIN000004614). The trial protocol was approved by the Institutional Review Boards of Yamaguchi University, Shimonoseki Medical Center and Kinki University, and the trial was conducted based on the ethical principles established in the Declaration of Helsinki. The patients were allocated to an LEM group or a placebo group by a block randomization method with central randomization. The anthracycline-based chemotherapy included any of the following: $5-\mathrm{FU}, 500 \mathrm{mg} / \mathrm{m}^{2}$; cyclophosphamide, $500 \mathrm{mg} / \mathrm{m}^{2}$; and epirubicin, $100 \mathrm{mg} / \mathrm{m}^{2}$ (FEC100 regimen), cyclophosphamide, $600 \mathrm{mg} / \mathrm{m}^{2}$ and epirubicin, $90 \mathrm{mg} / \mathrm{m}^{2}$ (EC regimen), 5 -FU, $500 \mathrm{mg} / \mathrm{m}^{2}$; cyclophosphamide, $500 \mathrm{mg} / \mathrm{m}^{2}$; and doxorubicin/pirarubicin, $50 \mathrm{mg} / \mathrm{m}^{2}$ (FAC regimen), or cyclophosphamide, $600 \mathrm{mg} / \mathrm{m}^{2}$; and doxorubicin/pirarubicin, $60 \mathrm{mg} / \mathrm{m}^{2}$ (AC regimen). The inclusion/non-inclusion of 5-FU in the regimens was used as an allocation factor. For each of these regimens, 3 weeks comprised a single course and patients received the chemotherapies on day 1 in each course. During the first 2 courses, LEM (daily dose of $1,800 \mathrm{mg}$ ) or placebo tablets were ingested daily over the 3 weeks of both courses, for a total of 6 weeks.

Measurement. In all the tests, measurements were made at five different time points: On day 1 (prior to administration of chemotherapy), day 8 (after administration of chemotherapy for 1 week) and day 22 (after administration of chemotherapy for 3 weeks and prior to administration of chemotherapy in the second course) in the first course, and on day 8 (after administration of chemotherapy for 1 week) and day 22 (after administration of chemotherapy for 3 weeks) in the second course.

Asurvey ofQOL wasmeasured by the Functional Assessment of Cancer Therapy (FACT) for patients receiving Biological Response Modifiers (BRM), version 4 (FACIT.org) (25), and was evaluated from scores on the basis of the Scoring \& Interpretation Materials of the questionnaire provided by FACIT.org. AEs were measured by the Common Terminology Criteria for Adverse Events (CTCAE) v.4.0-Japan Clinical Oncology Group edition (http://ctep.cancer.gov/protocol Development/electronic_applications/ctc.htm\#ctc_40).

Among immune parameters, the Th1/Th2 balance was measured by flow cytometric analysis of the proportion of interleukin (IL)-4-positive and interferon (IFN)- $\gamma$-positive 
cells among CD4-positive lymphocytes in the peripheral blood. Cell surface CD4 was detected with anti-CD4 PC-5-labeled antibody (dilution, 1:20; catalog no. 566004, BD Japan, Tokyo, Japan). Intracellular IL-4 and IFN- $\gamma$ were detected with anti-IL-4 phycoerythrin (PE)-labeled antibody (dilution, 1:20; catalog no. 340451) and anti-IFN- $\gamma$ fluorescein isothiocyanate (FITC)-labeled antibodies (dilution, 1:20; catalog no. 340449, BD Japan), respectively. Regulatory $\mathrm{T}$ cells (Tregs) were measured by flow cytometric analysis of the proportion of forkhead box p3 (FoxP3)-positive cells among CD4-positive lymphocytes in the peripheral blood. Cell surface CD4 and CD25 were detected using anti-CD4 FITC-labeled and anti-CD25 PE-Cy5.5-labeled (dilution, 1:20; catalog no. 560503, BD Japan) antibodies, respectively. In addition, intracellular FoxP3 was detected using anti-FoxP3 PE-labeled antibody (dilution, 1:20; catalog no. 560046, BD Japan).

NK cell activity was measured using a ${ }^{51} \mathrm{Cr}$ release assay. Peripheral blood mononuclear cells (PBMCs) were isolated using Ficoll solution. The isolated PBMCs were rinsed in RPMI-1640 medium containing $10 \%$ fetal bovine serum (FBS), adjusted to $1 \times 10^{6}$ cells $/ \mathrm{ml}$, and used as effector cells K562 cells (DS Pharma Biomedical Co. Ltd., Osaka, Japan), a human immortalized myelogenous leukemia cell line, were used for adjusting the concentration of the target cells. A total of $100 \mu \mathrm{Ci}$ of ${ }^{51} \mathrm{Cr}$ were added to the $\mathrm{K} 562$ cells and the cells were then cultured for $1 \mathrm{~h}$ at $37^{\circ} \mathrm{C}$. After rinsing twice with phosphate-buffered saline, the cell number was adjusted to $1 \times 10^{6}$ cells $/ \mathrm{ml}$ in RPMI-1640 medium containing $10 \% \mathrm{FBS}$, and these cells were used as the target cells. A mixed culture of effector and target cells was performed for $3.5 \mathrm{~h}$ at an effector/target $(\mathrm{E} / \mathrm{T})$ ratio of 20 , after which time ${ }^{51} \mathrm{Cr}$ released from dead target cells was measured using a $\gamma$-scintillation counter. In the maximum dissociation control, $1 \mathrm{~N}-\mathrm{HCl}$ was added in the place of effector cells, and ${ }^{51} \mathrm{Cr}$ released from dead target cells was measured with a $\gamma$-scintillation counter. In the spontaneous dissociation control, RPMI-1640 medium containing $10 \%$ FBS was added in the place of effector cells. The activity of NK cells was calculated as follows: Volume of released ${ }^{51} \mathrm{Cr}$ at the time of effector cell addition minus that in the spontaneous dissociation control/volume of released ${ }^{51} \mathrm{Cr}$ in the maximum dissociation control minus that in the spontaneous dissociation control.

Statistical analysis. Measurements are shown as means \pm standard error. Variation within each group was assayed by the Kruskal-Wallis test with the Steel test for QOL scores, and by repeated one-way analysis of variance with Bonferroni's correction for immune parameters.

\section{Results}

Patients. Between 2011 and 2014, 47 female breast cancer patients with a performance status of 0 or 1 (determined according to the Eastern Cooperative Oncology Group performance status in the Common Toxicity Criteria, v.2.0 (http://ctep.cancer.gov/protocolDevelopment/electronic_ app-lications/docs/ctcv20_4-30-992.pdf) were enrolled in the present study by the Department of Digestive Surgery and Surgical Oncology at Yamaguchi University, the
Table I. Patient characteristics.

\begin{tabular}{|c|c|c|}
\hline & LEM, n & Placebo, $\mathrm{n}$ \\
\hline Entry & 23 & 24 \\
\hline \multicolumn{3}{|l|}{ Age, years } \\
\hline Mean (range) & $62.0(31-85)$ & $57.5(42-77)$ \\
\hline \multicolumn{3}{|l|}{ Tumor stage } \\
\hline 0 & 0 & 0 \\
\hline 1 & 6 & 9 \\
\hline 2 & 14 & 12 \\
\hline 3 & 3 & 3 \\
\hline 4 & 0 & 0 \\
\hline \multicolumn{3}{|l|}{ T stage } \\
\hline Tis & 0 & 0 \\
\hline T0 & 2 & 0 \\
\hline $\mathrm{T} 1$ & 10 & 10 \\
\hline $\mathrm{T} 2$ & 10 & 12 \\
\hline $\mathrm{T} 3$ & 1 & 0 \\
\hline $\mathrm{T} 4$ & 0 & 0 \\
\hline Unknown & 0 & 2 \\
\hline \multicolumn{3}{|l|}{$\mathrm{N}$ stage } \\
\hline N0 & 11 & 16 \\
\hline N1 & 10 & 5 \\
\hline $\mathrm{N} 2$ & 2 & 2 \\
\hline N3 & 0 & 1 \\
\hline \multicolumn{3}{|l|}{ Treatments } \\
\hline FEC/FAC therapy & 10 & 12 \\
\hline $\mathrm{AC} / \mathrm{EC}$ therapy & 13 & 12 \\
\hline
\end{tabular}

LEM, Lentinula edodes mycelia extract; FEC, 5-FU + cyclophosphamide + epirubicin; FAC, 5-FU + cyclophosphamide + doxorubicin/pirarubicin; AC, cyclophosphamide + doxorubicin/pirarubicin; EC, cyclophosphamide + epirubicin; 5-FU, 5- fluorouracil.

Shimonoseki Medical Center of Japan Community Health Care Organization, and the Departments of Surgery and Medical Oncology at Kinki University Hospital, and scheduled to receive postoperative adjuvant anthracycline-based chemotherapy. The patients were allocated to the LEM group $(n=23)$ or the placebo group $(n=24)$ by a block randomization method. The patient background information is summarized in Table I. The age of the patients in the LEM group ranged from 31 to 85 years (median, 62 years) and, although none of the patients had distant metastasis, 12 were positive for lymph node metastasis. The age of the patients in the placebo group ranged from 42 to 77 years (median, 57.5 years) and, although none of the cases had distant metastasis, 8 patients were positive for lymph node metastasis.

$Q O L$. Patients with missing QOL score data for $\geq 2$ time points were excluded, leaving 22 patients in the LEM group and 21 patients in the placebo group for analysis of the QOL values. The changes in the QOL scores of FACT-BRM, FACT-general $(G)$, physical well-being (PWB) and functional well-being (FWB) during the study in graphic form 


\section{$\mathbf{A} \quad-$ LEM group $\quad-\square-$ Placebo group $\quad \mathbf{B}$}

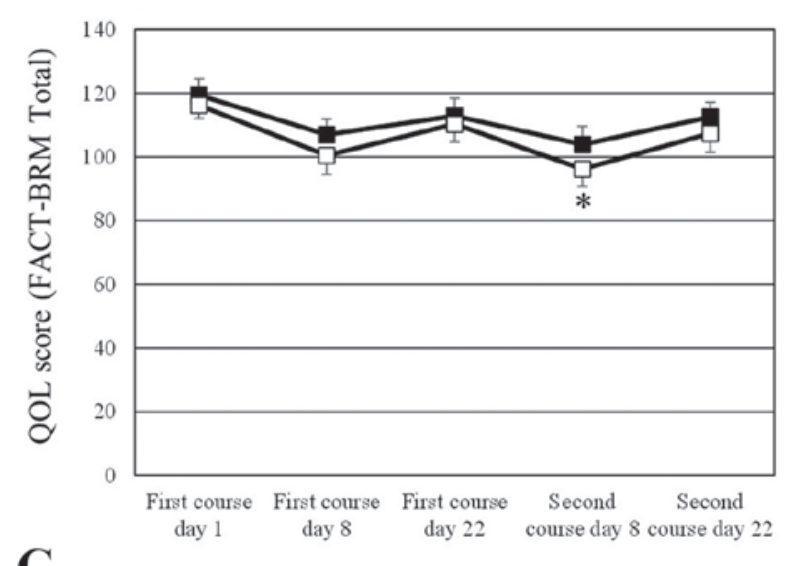

C
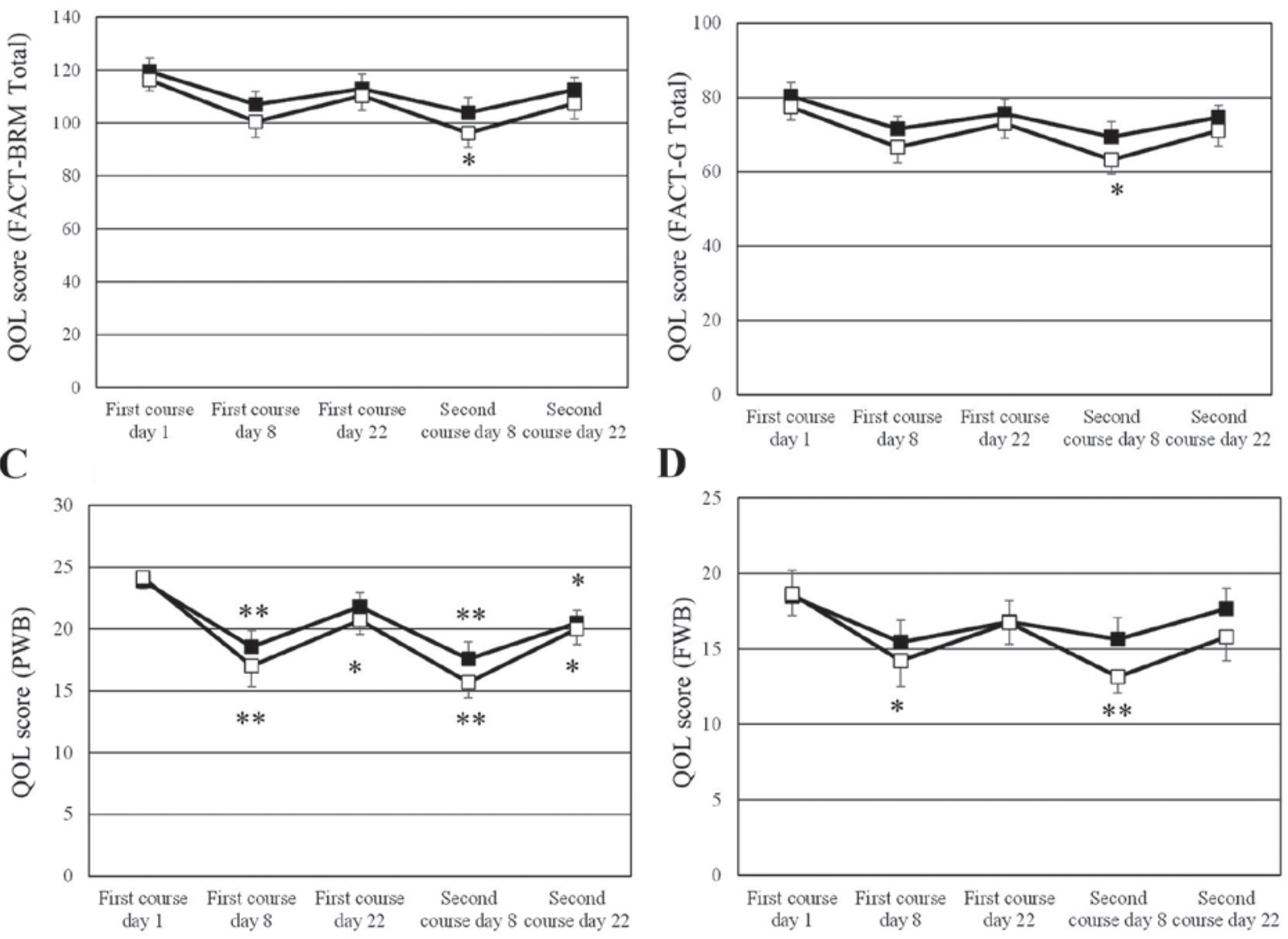

Figure 1. QOL scores over the course of the study. QOL was measured by the Functional Assessment of Cancer Therapy for patients receiving Biological Response Modifiers (FACT-BRM) version 4, and was evaluated from scores on the questionnaire. The measurement values shown are means \pm standard error. Variation within each group was assayed by the Kruskal-Wallis test with the Steel test vs. day 1 of the first course. " $\mathrm{P}<0.05$; ${ }^{* *} \mathrm{P}<0.01$. (A) Total scores, (B) FACT-G total scores, (C) PWB scores, (D) FWB scores. QOL, quality of life; LEM, Lentinula edodes mycelia extract; PWB, physical well-being; FWB, functional well-being; FACT-G, Functional Assessment of Cancer Therapy general scale.

are shown in Fig. 1. The numerical changes in all the QOL scores tested are shown in Table II. The FACT-BRM total score and the FACT-G total score had significantly decreased from the baseline scores in the placebo group on day 8 of the second course of chemotherapy, whereas the scores had not decreased in the LEM group. The FACT-BRM Trial Outcome Index had decreased from the baseline score in the placebo group on day 8 of both the first and the second courses of chemotherapy, whereas in the LEM group the score was only decreased on day 8 of the second course of chemotherapy. In the subscales, the PWB score had significantly decreased from the baseline value in both groups on day 8 of both courses of chemotherapy. In the LEM group, the decreased PWB score on day 8 of the first course of chemotherapy had recovered on day 22 of that course, while the score in the placebo group had not recovered. The FWB score had significantly decreased from the baseline value in the placebo group on day 8 of both courses of chemotherapy, whereas the score did not decrease in the LEM group during either of the chemotherapy courses. The BRM physical subscale (BRMP) score had significantly decreased from the baseline value in the placebo group on day 8 of both courses of chemotherapy, while the score was not decreased on day 8 of the first course of chemotherapy in the LEM group. No significant changes were observed in any of the other subscale scores during either of the two chemotherapy courses.

Toxicity. No AEs due to LEM occurred during this study. Table III summarizes the grade $\geq 3$ AEs (CTCAE v.4) that occurred due to anthracycline-based chemotherapy. Of these AEs, the following occurred in the LEM group: Anorexia $(n=1)$, nausea $(n=1)$, febrile neutropenia $(n=1)$ and decrease in neutrophil/lymphocyte ratio $(n=5)$. The AEs in the placebo group were as follows: Febrile neutropenia $(n=2)$, decrease in neutrophil/lymphocyte ratio $(\mathrm{n}=4)$, hand-foot skin reaction $(n=1)$ and erythroderma $(n=1)$. No differences in AEs were observed between the two groups. However, the 2 placebo group patients with febrile neutropenia were unable to complete the second course of treatment and dropped out of the study. There were no dropouts in the LEM group.

Immune parameters. Patients with missing immune parameter data for $\geq 2$ time points were excluded, leaving 23 patients in the LEM group and 19 patients in the placebo group for analysis of the immune parameters. The data regarding the changes in immune parameters during the study are summarized in Table IV. NK cell activity significantly decreased from the baseline value in both groups on day 8 of both the first and second 
Table II. Effect of LEM vs. placebo on QOL scores during the study course.

\begin{tabular}{|c|c|c|c|c|c|c|c|}
\hline \multirow[b]{2}{*}{ Scores } & \multirow[b]{2}{*}{ Groups } & \multirow[b]{2}{*}{ Subject no. } & \multicolumn{3}{|c|}{ First course } & \multicolumn{2}{|c|}{ Second course } \\
\hline & & & Day 1 & Day 8 & Day 22 & Day 8 & Day 22 \\
\hline \multirow[t]{2}{*}{ FACT-BRM total score } & LEM & 21 & $119.5 \pm 5.1$ & $107.0 \pm 4.9$ & $112.9 \pm 5.5$ & $103.9 \pm 5.7$ & $112.6 \pm 4.6$ \\
\hline & Placebo & 21 & $116.2 \pm 4.1$ & $100.5 \pm 5.9$ & $110.4 \pm 5.6$ & $96.2 \pm 5.4^{\mathrm{a}}$ & $107.4 \pm 5.9$ \\
\hline \multirow[t]{2}{*}{ FACT-G total score } & LEM & 21 & $80.4 \pm 3.6$ & $71.6 \pm 3.4$ & $75.6 \pm 3.8$ & $69.4 \pm 4.1$ & $74.7 \pm 3.2$ \\
\hline & Placebo & 21 & $77.4 \pm 3.5$ & $66.6 \pm 4.2$ & $73.0 \pm 4.0$ & $63.2 \pm 3.7^{\mathrm{a}}$ & $71.1 \pm 4.2$ \\
\hline \multirow[t]{2}{*}{ FACT-BRM TOI } & LEM & 21 & $81.4 \pm 3.7$ & $69.4 \pm 3.9$ & $75.9 \pm 4.0$ & $67.7 \pm 4.1^{\mathrm{a}}$ & $76.0 \pm 3.5$ \\
\hline & Placebo & 21 & $81.6 \pm 2.4$ & $65.1 \pm 4.3^{b}$ & $74.8 \pm 3.8$ & $61.8 \pm 3.8^{\mathrm{b}}$ & $72.1 \pm 4.3$ \\
\hline \multirow[t]{2}{*}{ PWB } & LEM & 22 & $23.9 \pm 0.8$ & $18.6 \pm 1.3^{\mathrm{b}}$ & $21.8 \pm 1.1$ & $17.6 \pm 1.4^{\mathrm{b}}$ & $20.5 \pm 1.0^{\mathrm{a}}$ \\
\hline & Placebo & 21 & $24.1 \pm 0.9$ & $17.0 \pm 1.7^{\mathrm{b}}$ & $20.7 \pm 1.2^{\mathrm{a}}$ & $15.7 \pm 1.3^{\mathrm{b}}$ & $20.0 \pm 1.3^{\mathrm{a}}$ \\
\hline \multirow[t]{2}{*}{ SWB } & LEM & 21 & $20.8 \pm 1.1$ & $20.6 \pm 1.3$ & $20.5 \pm 1.3$ & $20.1 \pm 1.3$ & $19.6 \pm 1.3$ \\
\hline & Placebo & 21 & $19.4 \pm 1.6$ & $20.2 \pm 1.5$ & $19.0 \pm 1.7$ & $19.6 \pm 1.5$ & $19.0 \pm 1.6$ \\
\hline \multirow[t]{2}{*}{ EWB } & LEM & 22 & $17.3 \pm 1.0$ & $17.0 \pm 1.1$ & $16.5 \pm 1.2$ & $16.1 \pm 1.4$ & $16.9 \pm 1.1$ \\
\hline & Placebo & 21 & $15.3 \pm 1.1$ & $15.2 \pm 1.2$ & $16.6 \pm 0.9$ & $14.8 \pm 1.1$ & $16.3 \pm 1.0$ \\
\hline \multirow[t]{2}{*}{ FWB } & LEM & 22 & $18.5 \pm 1.7$ & $15.4 \pm 1.5$ & $16.8 \pm 1.4$ & $15.6 \pm 1.4$ & $17.7 \pm 1.4$ \\
\hline & Placebo & 21 & $18.6 \pm 1.4$ & $14.2 \pm 1.7^{\mathrm{a}}$ & $16.7 \pm 1.4$ & $13.1 \pm 1.1^{b}$ & $15.8 \pm 1.6$ \\
\hline \multirow[t]{2}{*}{ BRMP } & LEM & 21 & $22.5 \pm 1.0$ & $20.4 \pm 0.7$ & $20.8 \pm 0.9$ & $18.7 \pm 1.1^{\mathrm{a}}$ & $20.8 \pm 0.8$ \\
\hline & Placebo & 21 & $22.1 \pm 1.0$ & $19.3 \pm 1.0^{\mathrm{a}}$ & $21.5 \pm 0.8$ & $18.8 \pm 1.0^{\mathrm{a}}$ & $20.9 \pm 1.1$ \\
\hline \multirow[t]{2}{*}{ BRMCE } & LEM & 21 & $16.6 \pm 1.1$ & $15.0 \pm 1.2$ & $16.5 \pm 1.3$ & $15.8 \pm 1.0$ & $17.1 \pm 1.0$ \\
\hline & Placebo & 21 & $16.7 \pm 1.0$ & $14.6 \pm 1.2$ & $15.9 \pm 1.2$ & $14.2 \pm 1.3$ & $15.4 \pm 1.2$ \\
\hline
\end{tabular}

Measurement values are shown as mean \pm standard error. Variation within each group was assayed by the Kruskal-Wallis test with the Steel test vs. day 1 of the first course. ${ }^{\mathrm{a}} \mathrm{P}<0.05$; ${ }^{\mathrm{b}} \mathrm{P}<0.01$. LEM, Lentinula edodes mycelia extract; QOL, quality of life; FACT, Functional Assessment of Cancer Therapy; G, general scale; TOI, Trial Outcome Index; PWB, physical well-being; SWB, social/family well-being; EWB, emotional well-being; FWB, functional well-being; BRMP, BRM physical subscale; BRMCE, BRM cognitive/emotional subscale; BRM, biological response modifier.

Table III. Adverse events with LEM vs. placebo treatment.

\begin{tabular}{lcc}
\hline Adverse events, $\mathrm{n}$ & LEM & Placebo \\
\hline Anorexia & 1 & 0 \\
Nausea & 1 & 0 \\
Febrile neutropenia & 1 & 2 \\
Neutrophil/lymphocyte decrease & 5 & 4 \\
Hand-foot skin reaction & 0 & 1 \\
Erythroderma & 0 & 1 \\
\hline
\end{tabular}

LEM, Lentinula edodes mycelia extract.

courses of chemotherapy. In the LEM group, NK cell activity also decreased on day 22 of the second course. The proportion of Tregs in the peripheral blood $\mathrm{CD}^{+}$cells $\left(\mathrm{FoxP} 3^{+} \mathrm{CD} 25^{+} / \mathrm{CD} 4^{+}\right.$) was significantly decreased in the LEM group on day 8 of the first chemotherapy course. In the placebo group, that proportion was significantly increased from the baseline value on day 22 of both chemotherapy courses. Even in the LEM group, the proportion was significantly increased from the baseline value on day 22 of the second chemotherapy course, but the degree of that increase tended to be lower compared with that in the placebo group (Fig. 2). The Th1/Th2 balance exhibited no significant changes in either the LEM or the placebo group.

\section{Discussion}

Anthracycline-based chemotherapies are effective and are widely used as the main postoperative adjuvant chemotherapy for patients with early-stage breast cancer (1-3). However, those treatments are accompanied by a high incidence of nausea and vomiting $(4,5)$, severely compromising the patients' QOL. The use of 5HT3 receptor antagonists has become commonplace in recent years, enabling a certain level of control of these symptoms. However, fatigue and decreased physical QOL remain challenging issues in breast cancer patients receiving postoperative adjuvant chemotherapy, and methods for alleviating these symptoms are needed (9). In our earlier single-group open study, in which LEM was coadministered to breast cancer patients who were treated with the FEC75 regimen, the QOL-ACD (24) was used to evaluate the effect of LEM on the patients' QOL. The results indicated that coadministration of LEM was effective in improving the patients' physical scale in response to the FEC75 regimen. LEM is a BRM agent; thus, in the present study, the QOL was evaluated using the FACT-BRM (25), which was developed for assessing the effects of BRM on the QOL of cancer patients.

The FACT-BRM total score was significantly decreased from the baseline score in the placebo group on day 8 of the second course of chemotherapy, whereas in the LEM group 
Table IV. Effect of LEM vs. placebo on immunological parameters during the study course.

\begin{tabular}{|c|c|c|c|c|c|c|c|}
\hline \multirow[b]{2}{*}{ Parameters } & \multirow[b]{2}{*}{ Groups } & \multirow[b]{2}{*}{ Subject no. } & \multicolumn{3}{|c|}{ First course } & \multicolumn{2}{|c|}{ Second course } \\
\hline & & & Day 1 & Day 8 & Day 22 & Day 8 & Day 22 \\
\hline \multirow[t]{2}{*}{ NK cell activity (\%) } & LEM & 23 & $32.5 \pm 3.7$ & $19.1 \pm 2.9^{b}$ & $23.4 \pm 2.4^{\mathrm{b}}$ & $13.6 \pm 1.7^{\mathrm{b}}$ & $25.2 \pm 2.7^{\mathrm{a}}$ \\
\hline & Placebo & 19 & $27.4 \pm 3.1$ & $16.2 \pm 1.9^{b}$ & $20.4 \pm 1.8$ & $10.8 \pm 2.2^{b}$ & $23.2 \pm 2.9$ \\
\hline \multirow{4}{*}{$\begin{array}{l}\text { Treg }\left(\mathrm{Foxp}^{+} \mathrm{CD} 25^{+} / \mathrm{CD}^{+}\right. \\
\text {cells) }(\%) \\
\text { Th1/Th2 balance }\left(\mathrm{IFN}-\gamma^{+} / \mathrm{IL}-4^{+}\right. \\
\left.\text {in } \mathrm{CD} 4^{+} \text {cells }\right)\end{array}$} & LEM & 23 & $6.2 \pm 0.3$ & $5.2 \pm 0.3^{\mathrm{a}}$ & $6.6 \pm 0.4$ & $5.5 \pm 0.2$ & $7.3 \pm 0.4^{\mathrm{b}}$ \\
\hline & Placebo & 19 & $5.8 \pm 0.3$ & $5.1 \pm 0.3$ & $6.6 \pm 0.4^{\mathrm{a}}$ & $5.6 \pm 0.4$ & $7.6 \pm 0.4^{\mathrm{b}}$ \\
\hline & LEM & 23 & $10.1 \pm 1.5$ & $8.7 \pm 1.3$ & $9.2 \pm 1.1$ & $7.7 \pm 0.9$ & $8.1 \pm 1.0$ \\
\hline & Placebo & 19 & $10.3 \pm 1.4$ & $9.5 \pm 1.2$ & $10.1 \pm 1.1$ & $8.7 \pm 1.1$ & $10.5 \pm 1.3$ \\
\hline
\end{tabular}

Measurement values are shown as means \pm standard error. Variation within each group was assayed by repeated one-way analysis of variance with Bonferroni's correction vs. day 1 of the first course. ${ }^{a} \mathrm{P}<0.05$; ${ }^{\mathrm{b}} \mathrm{P}<0.01$. LEM, Lentinula edodes mycelia extract; NK, natural killer; Treg, regulatory T cells; IFN, interferon; IL, interleukin.

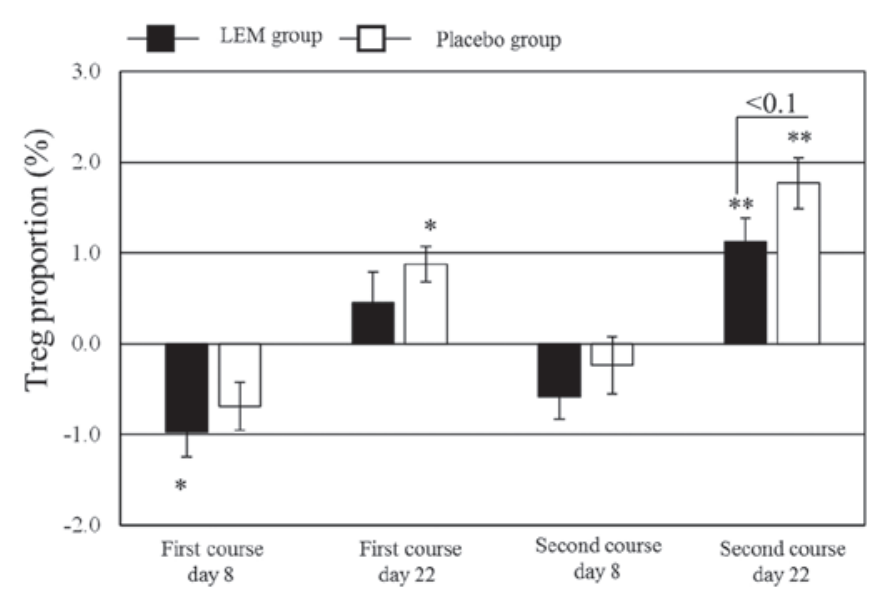

Figure 2. Regulatory T cells (Tregs) in CD4-positive cells in peripheral blood over the course of the study. The proportion of CD25-positive FoxP3-positive cells in CD4-positive cells was measured using flow cytometry. The figure shows the change in the value from the baseline (first course, day 1, prior to drug administration). The measurement values are shown as means \pm standard error. Black bars, LEM group; white bars, placebo group Variation within each group was assayed by repeated one-way analysis of variance with Bonferroni's correction vs. day 1 of the first course. ${ }^{*} \mathrm{P}<0.05$; ${ }^{* *} \mathrm{P}<0.01$. Variation between the two groups was assayed by an unpaired t-test. LEM, Lentinula edodes mycelia extract; FoxP3, forkhead box p3.

this score was not decreased. The placebo group also exhibited significantly reduced scores for the PWB, BRMP and FWB subscales on day 8 of both the first and second courses of chemotherapy in comparison with the baseline scores, and the PWB had not recovered to the baseline score on day 22 of both courses. The PWB questions include one regarding nausea and vomiting. In the present study, only 1 patient experienced grade $\geq 3$ severe nausea and vomiting, and the nausea and vomiting were controlled to a certain extent by the use of 5HT3 receptor antagonists. However, the PWB scores had not recovered in either course on day 22 in the placebo group, suggesting that the patients' physical QOL must be improved. In the LEM group, the PWB scores were decreased on day 8 of both chemotherapy courses compared with their baseline scores, as well as compared with the placebo group, but they had recovered by day 22 of the first course. Furthermore, unlike in the placebo group, the BRMP score, which includes questions regarding fatigue, was not decreased on day 8 of the first chemotherapy course in the LEM group. Moreover, the FWB score was not decreased in the LEM group. These results indicate that coadministration of LEM with anthracycline-based chemotherapies was effective in alleviating decreases in the QOL, particularly regarding the patients' physical condition and functional activity.

An attempt to ameliorate the chemotherapy-induced fatigue and decrease in the physical QOL in breast cancer patients by means of resistance exercise was reported (26), but no reliable protocols have been established to date. LEM was also reported to improve the QOL in breast cancer patients who receive postoperative hormone therapy (22). Combined with the results of the present study, these data suggest that coadministration of LEM may be a useful method for better preserving the QOL of postoperative breast cancer patients undergoing a broad range of drug therapies.

The host immune function of breast cancer patients was reported to affect the prognosis of such patients receiving anticancer chemotherapy (27). The present study investigated the mechanism through which anthracycline-based chemotherapies affect NK cell activity, Tregs and the Th1/Th2 balance, and evaluated the effect of coadministered LEM on these immune parameters (Table IV). In our earlier single-group open study on postoperative breast cancer patients who were treated with the FEC75 regimen, NK cell activity was found to be decreased after 1 week of chemotherapy, but coadministration of LEM inhibited that decrease. However, in the present study, NK cell activity was significantly reduced on day 8 of both the first and second chemotherapy courses in both the placebo and the LEM groups, and we were unable to demonstrate that coadministration of LEM prevented that decrease. Further investigation into the effect of coadministration of LEM on NK cell activity is required. In the present study, no significant change was observed in the Th1/Th2 balance in either of the two groups, which is in agreement with the results of our earlier study. Th1/Th2 balance analysis is a T-cell subset analysis that evaluates the proportion of IFN- $\gamma$-producing and IL-4-producing cells among CD4 ${ }^{+}$ lymphocytes. In recent years, it has become widely recognized 
that, for induction of anticancer immunity, the proportion of Tregs (FoxP3 ${ }^{+} / \mathrm{CD} 25^{+}$cells) in $\mathrm{CD}^{+}$lymphocytes provides a more important index of the tumor immune status compared with the Th1/Th2 balance. That is, an increase in Tregs in the tumor-bearing state is known to result in a decrease in tumor immune function (28-30). In the present study, the proportion of Tregs was significantly increased over the baseline value in the placebo group on day 22 of both the first and second chemotherapy courses, while the lymphocyte count was found to be decreased on day 8 of both courses. Therefore, it may be surmised that the lymphocyte count decreased transiently due to the anthracycline-based chemotherapy, and then, during the ensuing recovery process, the immunosuppressive Tregs recovered earlier among the subsets of $\mathrm{CD}^{+}$cells. On the other hand, in the LEM group, the Treg proportion on day 8 of course 1 was significantly reduced compared with the baseline value, and this reduction was maintained on day 22 of course 1 . In addition, on day 22 of course 2 , as in the placebo group, the Treg proportion increased significantly compared with the baseline value, but the degree of that increase tended to be lower compared with the placebo group. In vivo, LEM mitigated the immunosuppression mediated by the tumor-bearing host's Tregs and induced antitumor immunity $(18,19)$. Moreover, in a study on patients receiving cancer immune-cell therapy, the increase in Tregs accompanying cancer progression was reported to be improved (31). It is known that certain chemotherapies may reduce Tregs (32), and low-dose cyclophosphamide, which is a drug used in anthracycline-based regimens, is known to somewhat selectively reduce Tregs $(33,34)$. In the present study, the dose of cyclophosphamide was not as low as in the former reports $(33,34)$, and it is perhaps for that reason that the total lymphocyte count appeared to be decreased non-selectively in both groups. In the LEM group, the data suggested that LEM may promote the reduction of Tregs on day 8 of course 1 and inhibit the increase in Tregs on day 22 of course 2 after the recovery process. With regard to the association between the prognosis of breast cancer patients and Tregs, a number of reports have verified the association between Tregs in the tumor-infiltrating lymphocyte population and prognosis. However, the association of Tregs with prognosis remains controversial, possibly due to variations in the tumor microenvironment $(33,35,36)$. With regard to the Treg proportion among $\mathrm{CD} 4^{+}$cells in the peripheral blood of breast cancer patients administered postoperative adjuvant therapy who were evaluated in this study, it was reported that this proportion decreases temporarily after surgery, and then rebounds after postoperative adjuvant chemotherapy (37). It may be hypothesized that, in breast cancer patients, suppression of immune function occurs following postoperative adjuvant therapy, similar to that occurring under tumor-bearing conditions. Based on the results of the present study, indicating that coadministration of LEM has the potential to improve the suppression of immune function that occurs following adjuvant chemotherapy, more detailed studies on this subject are anticipated in the future.

In conclusion, postoperative breast cancer patients who receive anthracycline-based chemotherapy experience decreased QOL and immune function. However, coadministration of LEM may ameliorate those decreases in QOL and immune function. Accordingly, oral LEM may prove to be a useful supportive care option for such postoperative breast cancer patients.

\section{Acknowledgements}

The present study received a research grant from the Osaka Cancer Research Foundation.

\section{References}

1. Group (EBCTCG): Effects of chemotherapy and hormonal therapy for early breast cancer on recurrence and 15-year survival: An overview of the randomised trials. Lancet 365 : 1687-1717, 2005.

2. French Adjuvant Study Group: Benefit of a high-dose epirubicin regimen in adjuvant chemotherapy for node-positive breast cancer patients with poor prognostic factors: 5-year follow-up results of French Adjuvant Study Group 05 randomized trial. J Clin Oncol 19: 602-611, 2001.

3. Turner N, Biganzoli L and Di Leo A: Continued value of adjuvant anthracyclines as treatment for early breast cancer. Lancet Oncol 16: e362-e369, 2015.

4. Riccardi A, Brugnatelli S, Giordano M, Danova M, Pugliese P, Tinelli C, Klersy C, Richetti A, Fava S, Nastasi G, et al: Myeloprotective effect of early primary granulocyte-colony stimulating factor during six courses of intensified 5-fluorouracil, epirubicin and cyclophosphamide (120FEC) chemotherapy for advanced breast cancer. Cooperative group of study and treatment of breast cancer. Tumori 84: 540-546, 1998

5. Baltali E, Günel N, Onat DA, Atahan IL, Akçali Z, Büyukünal E and Firat D: Neoadjuvant chemotherapy in locally advanced breast cancer: A preliminary report. Turkish oncology study group. Tumori 85: 483-487, 1999.

6. Takeuchi H, Saeki T, Aiba K, Tamura K, Aogi K, Eguchi K, Okita K, Kagami Y, Tanaka R, Nakagawa K, et al: Japanese society of clinical oncology clinical practice guidelines 2010 for antiemesis in oncology: Executive summary. Int J Clin Oncol 21: $1-12,2016$.

7. Ohzawa H, Miki A, Hozumi Y, Miyazaki C, Sagara Y, Tanaka Y, Shiba S, Joutoku H, Sakuragi M, Takehara M, et al: Comparison between the antiemetic effects of palonosetron and granisetron in breast cancer patients treated with anthracycline-based regimens. Oncol Lett 9: 119-124, 2015.

8. Rapoport BL, Jordan K, Boice JA, Taylor A, Brown C, Hardwick JS, Carides A, Webb T and Schmoll HJ: Aprepitant for the prevention of chemotherapy-induced nausea and vomiting associated with a broad range of moderately emetogenic chemotherapies and tumor types: A randomized, double-blind study. Support Care Cancer 18: 423-431, 2010.

9. Berger AM, Lockhart K and Agrawal S: Variability of patterns of fatigue and quality of life over time based on different breast cancer adjuvant chemotherapy regimens. Oncol Nurs Forum 36: $563-570,2009$

10. Kornblith AB, Lan L, Archer L, Partridge A, Kimmick G, Hudis C, Winer E, Casey R, Bennett S, Cohen HJ and Muss HB: Quality of life of older patients with early-stage breast cancer receiving adjuvant chemotherapy: A companion study to cancer and leukemia group B 49907. J Clin Oncol 29: 1022-1028, 2011.

11. Martín M, Lluch A, Seguí MA, Ruiz A, Ramos M, Adrover E, Rodriguez-Lescure A, Grosse R, Calvo L, Fernandez-Chacón C, et al: Toxicity and health-related quality of life in breast cancer patients receiving adjuvant docetaxel, doxorubicin, cyclophosphamide (TAC) or 5-fluorouracil, doxorubicin and cyclophosphamide (FAC): Impact of adding primary prophylactic granulocyte-colony stimulating factor to the TAC regimen. Ann Oncol 17: 1205-1212, 2006.

12. Apetoh L, Ghiringhelli F, Tesniere A, Obeid M, Ortiz C, Criollo A, Mignot G, Maiuri MC, Ullrich E, Saulnier P, et al: Toll-like receptor 4-dependent contribution of the immune system to anticancer chemotherapy and radiotherapy. Nat Med 13: 1050-1059, 2007.

13. Mozaffari F, Lindemalm C, Choudhury A, GranstamBjörneklett H, Helander I, Lekander M, Mikaelsson E, Nilsson B, Ojutkangas ML, Osterborg A, et al: NK-cell and T-cell functions in patients with breast cancer: effects of surgery and adjuvant chemo- and radiotherapy. Br J Cancer 97: 105-111, 2007. 
14. Kawanishi T, Ikeda-Dantsuji Y and Nagayama A: Effects of two basidiomycete species on interleukin 1 and interleukin 2 production by macrophage and $\mathrm{T}$ cell lines. Immunobiology 215 : 516-520, 2010

15. Sugano N, Hibino Y, Choji Y and Maeda H: Anticarcinogenic actions of water-soluble and alcohol-insoluble fractions from culture medium of Lentinus edodes mycelia. Cancer Lett 17: 109-114, 1982

16. Sugano N, Choji Y, Hibino Y, Yasumura S and Maeda H: Anticarcinogenic action of an alcohol-insoluble fraction (LAP1) from culture medium of Lentinus edodes mycelia. Cancer Lett 27: 1-6, 1985.

17. Liu M, Li J, Kong F, Lin J and Gao Y: Induction of immunomodulating cytokines by a new polysaccharide-peptide complex from culture mycelia of Lentinus edodes. Immunopharmacology 40 : 187-198, 1998

18. Tanaka K, Matsui Y, Ishikawa S, Kawanishi T and Harada M: Oral ingestion of Lentinula edodes mycelia extract can restore the antitumor $\mathrm{T}$ cell response of mice inoculated with colon-26 cells into the subserosal space of the cecum. Oncol Rep 27: 325-332, 2012

19. Tanaka K, Ishikawa S, Matsui Y, Tamesada M, Harashima N and Harada M: Oral ingestion of Lentinula edodes mycelia extract inhibits B16 melanoma growth via mitigation of regulatory $\mathrm{T}$ cell-mediated immunosuppression. Cancer Sci 102: 516-521, 2011.

20. Yamaguchi Y, Miyahara E and Hihara J: Efficacy and safety of orally administered Lentinula edodes mycelia extract for patients undergoing cancer chemotherapy: A pilot study. Am J Chin Med 39: 451-459, 2011.

21. Okuno K and Uno K: Efficacy of orally administered Lentinula edodes mycelia extract for advanced gastrointestinal cancer patients undergoing cancer chemotherapy: A pilot study. Asian Pac J Cancer Prev 12: 1671-1674, 2011.

22. Suzuki N, Takimoto Y, Suzuki R, Arai T, Uebaba K, Nakai M, Strong JM and Tokuda H: Efficacy of oral administration of Lentinula edodes mycelia extract for breast cancer patients undergoing postoperative hormone therapy. Asian Pac J Cancer Prev 14: 3469-3472, 2013

23. Nagashima Y, Maeda N, Yamamoto S, Yoshino S and Oka M: Evaluation of host quality of life and immune function in breast cancer patients treated with combination of adjuvant chemotherapy and oral administration of Lentinula edodes mycelia extract. Onco Targets Ther 6: 853-859, 2013.

24. Kurihara M, Shimizu H, Tsuboi K, Kobayashi K, Murakami M, Eguchi K and Shimozuma K: Development of quality of life questionnaire in Japan: Quality of life assessment of cancer patients receiving chemotherapy. Psychooncology 8: 355-363, 1999.

25. Bacik J, Mazumdar M, Murphy BA, Fairclough DL, Eremenco S, Mariani T, Motzer RJ and Cella D: The functional assessment of cancer therapy-BRM (FACT-BRM): A new tool for the assessment of quality of life in patients treated with biologic response modifiers. Qual Life Res 13: 137-154, 2004.

26. Schmidt ME, Wiskemann J, Armbrust P, Schneeweiss A, Ulrich CM and Steindorf K: Effects of resistance exercise on fatigue and quality of life in breast cancer patients undergoing adjuvant chemotherapy: A randomized controlled trial. Int J Cancer 137: 471-480, 2015.
27. Mozaffari F, Lindemalm C, Choudhury A, GranstamBjörneklett H, Lekander M, Nilsson B, Ojutkangas ML, Osterborg A, Bergkvist L and Mellstedt H: Systemic immune effects of adjuvant chemotherapy with 5-fluorouracil, epirubicin and cyclophosphamide and/or radiotherapy in breast cancer: A longitudinal study. Cancer Immunol Immunother 58: 111-120, 2009.

28. Sato E, Olson SH, Ahn J, Bundy B, Nishikawa H, Qian F, Jungbluth AA, Frosina D, Gnjatic S, Ambrosone C, et al: Intraepithelial CD8+ tumor-infiltrating lymphocytes and a high $\mathrm{CD} 8+$ +regulatory $\mathrm{T}$ cell ratio are associated with favorable prognosis in ovarian cancer. Proc Natl Acad Sci USA 102: 18538-18543, 2005.

29. Yamaguchi T and Sakaguchi S: Regulatory T cells in immune surveillance and treatment of cancer. Semin Cancer Biol 16: 115-123, 2006.

30. Curiel TJ, Coukos G, Zou L, Alvarez X, Cheng P, Mottram P, Evdemon-Hogan M, Conejo-Garcia JR, Zhang L, Burow M, et al: Specific recruitment of regulatory $\mathrm{T}$ cells in ovarian carcinoma fosters immune privilege and predicts reduced survival. Nat Med 10: 942-949, 2004

31. Tanigawa K, Ito Y, Sakai M and Kobayashi Y: Evaluation of quality of life and immune function in cancer patients receiving combined immunotherapy and oral administration of lentinula edodes mycelia extract. Gan To Kagaku Ryoho 39: 1779-1781, 2012 (In Japanese).

32. Maeda K, Hazama S, Tokuno K, Kan S, Maeda Y, Watanabe Y, Kamei R, Shindo Y, Maeda N, Yoshimura K, et al: Impact of chemotherapy for colorectal cancer on regulatory T-cells and tumor immunity. Anticancer Res 31: 4569-4574, 2011

33. Bates GJ, Fox SB, Han C, Leek RD, Garcia JF, Harris AL and Banham AH: Quantification of regulatory T cells enables the identification of high-risk breast cancer patients and those at risk of late relapse. J Clin Oncol 24: 5373-5380, 2006.

34. Ercolini AM, Ladle BH, Manning EA, Pfannenstiel LW, Armstrong TD, Machiels JP, Bieler JG, Emens LA, Reilly RT and Jaffee EM: Recruitment of latent pools of high-avidity CD8(+) $\mathrm{T}$ cells to the antitumor immune response. J Exp Med 201: 1591-1602, 2005.

35. Liu S, Foulkes WD, Leung S, Gao D, Lau S, Kos Z and Nielsen TO: Prognostic significance of FOXP3+ tumor-infiltrating lymphocytes in breast cancer depends on estrogen receptor and human epidermal grow th factor receptor-2 expression status and concurrent cytotoxic T-cell infiltration. Breast Cancer Res 16: 432, 2014.

36. Aruga T, Suzuki E, Saji S, Horiguchi S, Horiguchi K, Sekine S, Kitagawa D, Funata N, Toi M, Sugihara K and Kuroi K: A low number of tumor-infiltrating FOXP3-positive cells during primary systemic chemotherapy correlates with favorable anti-tumor response in patients with breast cancer. Oncol Rep 22: 273-278, 2009.

37. Rech AJ, Mick R, Kaplan DE, Chang KM, Domchek SM and Vonderheide RH: Homeostasis of peripheral FoxP3(+) CD4 (+) regulatory $\mathrm{T}$ cells in patients with early and late stage breast cancer. Cancer Immunol Immunother 59: 599-607, 2010. 